\title{
Influences of resonant wavelengths on performances of microcavity organic light-emitting devices
}

\author{
Chun-Liang Lin, Han-Chieh Chang, Kun-Cheng Tien, and Chung-Chih Wu ${ }^{a)}$ \\ Department of Electrical Engineering, National Taiwan University, Taipei, Taiwan 10617, Republic of \\ China; Graduate Institute of Electro-optical Engineering, National Taiwan University, Taipei, Taiwan 10617, \\ Republic of China; and Graduate Institute of Electronics Engineering, National Taiwan University, \\ Taipei, Taiwan 10617, Republic of China
}

(Received 14 October 2006; accepted 11 January 2007; published online 16 February 2007)

\begin{abstract}
In this letter, we investigate theoretically and experimentally the influences of resonant wavelengths on performances of microcavity organic light-emitting devices. Results show that by setting the normal-direction resonant wavelength around the peak wavelength of the intrinsic emission, one obtains the highest luminance enhancement along the normal direction and hardly detectable color shift with viewing angles, yet accompanied by highly directed emission. On the other hand, the highest enhancement $(\sim 1.4$ times $)$ in external quantum efficiencies and the most uniform brightness distribution are obtained by setting the normal-direction resonant wavelength of $20-40 \mathrm{~nm}$ longer than the peak wavelength of the intrinsic emission, yet with noticeable color shift over viewing angles. Due to the trade-offs between different emission characteristics in choosing the resonant wavelength, the exact design of microcavity devices would depend on actual applications. (C) 2007 American Institute of Physics. [DOI: 10.1063/1.2472541]
\end{abstract}

Incorporation of the microcavity structure into organic light-emitting devices (OLEDs) is often demonstrated to narrow emission spectra and to enhance the luminance. ${ }^{1-7}$ While microcavity OLEDs may be implemented by either lossless dielectric mirrors or metal mirrors, metal mirrors are considered more practical for real applications. ${ }^{1-8}$ In designing microcavity devices, three most important considerations are the mirror properties, the cavity length (indeed determined by the cavity resonant wavelength desired), and the location of emitters in the cavity. ${ }^{1,2,7}$ For microcavity OLEDs using two metal mirrors, previous studies have determined that high-reflection and low-loss mirrors are essential for obtaining luminance enhancement. ${ }^{7}$ Once the mirror structures are determined, the other cavity geometries are mainly determined by the cavity resonant wavelength. For devices containing narrow-band emitters, to obtain optimal light output, it is intuitive to set the resonant wavelength of the microcavity at the peak wavelength of the intrinsic emission and subsequently locate the emitters around a common antinode of both mirror electrodes (i.e., the emitter-to-mirror round-trip phase change equals to integral multiples of $2 \pi){ }^{1,2,7}$ Yet for most cases of OLEDs, the intrinsic emission spectra of emitters are so broad (typically $>50 \mathrm{~nm}$ ) that it becomes less obvious in the broad spectra to set the resonant wavelength for performance optimization. In this letter, we investigate theoretically and experimentally the influences of the resonant wavelength on characteristics of microcavity OLEDs having two metal mirrors.

The microcavity OLEDs under investigation are based on the fluorescent green emitter C545T, whose intrinsic emission shows a peak around $521 \mathrm{~nm}\left(\lambda_{\mathrm{PL}}\right)$ and a full width at half maximum of $\sim 60 \mathrm{~nm}$. ${ }^{7,9}$ The devices have the structure of glass/Ag $(80 \mathrm{~nm}) / \mathrm{m}$-MTDATA: $\mathrm{F}_{4}$-TCNQ (2 wt. $\left.\%, x \mathrm{~nm}\right) / \alpha$-NPD $(20 \mathrm{~nm}) / \mathrm{Alq}_{3}: \mathrm{C} 545 \mathrm{~T}$ $(1$ wt. $\%, 20 \mathrm{~nm}) / \mathrm{Alq}_{3}(y \mathrm{~nm}) / \mathrm{LiF}(0.5 \mathrm{~nm}) / \mathrm{Al}(1 \mathrm{~nm}) / \mathrm{Ag}$

\footnotetext{
a) Author to whom correspondence should be addressed; electronic mail:
} chungwu@cc.ee.ntu.edu.tw
$(22 \mathrm{~nm}) / \mathrm{Alq}_{3}(70 \mathrm{~nm})$, which adopts the configuration with the high-reflectivity $\mathrm{Ag}(80 \mathrm{~nm})$ as the bottom anode and the semitransparent $\mathrm{Ag}(22 \mathrm{~nm})$ as the top cathode. According to previous studies of microcavity OLEDs, for maximizing the optical output, the thin Ag is further capped with a $70 \mathrm{~nm}$ high-index organic layer $\left(\mathrm{Alq}_{3}, n \sim 1.74\right)$ to achieve both high reflection $(\sim 55 \%$, seen from the inside of the device) and low absorption $(\sim 8 \%)$ for major emission wavelengths of $\mathrm{C}_{545 \mathrm{~T}^{4-9}}$ (as shown in Fig. 1). The organic multilayer structure on top of the $\mathrm{Ag}$ anode in sequence consists of 4, 4', 4"'-tris(3-methylphenylphenylamino) triphenylamine (m-MTDATA) doped with 2 wt. \% of tetrafluorotetracyanoquinodimethane $\left(\mathrm{F}_{4}\right.$-TCNQ) as the $p$-doped hole-injection layer, ${ }^{7,10} \alpha$-naphthylphenylbiphenyl diamine ( $\alpha$-NPD, as the hole-transport layer, ${ }^{7,9}$ tris-(8-hydroxyquinoline) aluminum $\left(\mathrm{Alq}_{3}\right)$ doped with C545T as the emitting layer, $5,7,9$ and undoped $\mathrm{Alq}_{3}$ as the electron-transport layer. ${ }^{5,7,9}$ Ultrathin layers of $\mathrm{LiF}$ and $\mathrm{Al}(1 \mathrm{~nm})$ serve as the electron-injecting contact. $^{5,7,9}$

In this device configuration, the thicknesses of m-MTDATA: $\mathrm{F}_{4}$-TCNQ and $\mathrm{Alq}_{3}$ are varied to adjust the

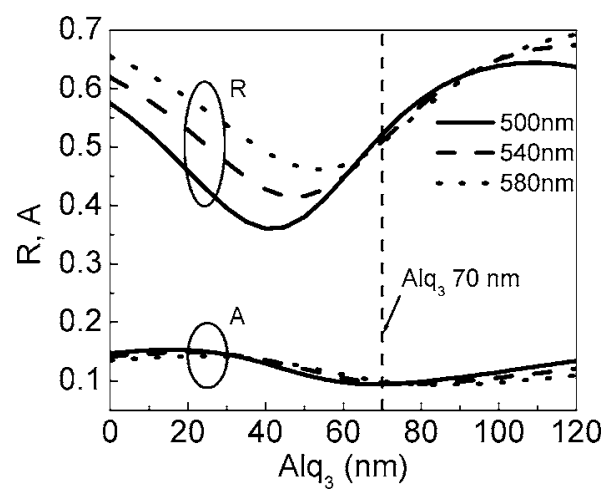

FIG. 1. Calculated reflectance $(R)$ and absorptance $(A)$ (seen from the interior of the device) of the [thin $\mathrm{Ag}(22 \mathrm{~nm}) / \mathrm{Alq}_{3}$ ] composite mirror as a function of the $\mathrm{Alq}_{3}$ thickness for various wavelengths. 
resonant wavelength (at the lowest resonance mode) and the emitter location (according to the antinode condition of the resonant wavelength). In this study, microcavity OLEDs with five different normal-direction resonant wavelengths $\lambda_{R}\left(0^{\circ}\right)$ of 500 (device B), 515 (device C), 545 (device D), 560 (device E), and $590 \mathrm{~nm}$ (device F) were fabricated and characterized using the (MTDATA: $\mathrm{F}_{4}$-TCNQ, $\mathrm{Alq}_{3}$ ) thicknesses $=(25,29), \quad(27,31),(32,35), \quad(35,37)$, and $(40,41) \mathrm{nm}$, respectively. Since the variation of the thickness of the electron-transport layer $\mathrm{Alq}_{3}$ is only $\sim 10 \mathrm{~nm}$, it shall not significantly alter the scenario of carrier injection and transport. Thus, the more complicated n-doped electron transport layer is not used here as we did in a previous work. ${ }^{11}$ For comparison, a nearly optimized conventional bottom-emitting OLED (device A) with the structure of glass/ITO $(120 \mathrm{~nm}) / \mathrm{m}-\mathrm{MTDATA}: \mathrm{F}_{4^{-}}$ TCNQ $\quad(2$ wt. $\%, 30 \mathrm{~nm}) / \alpha$-NPD $\quad(20 \mathrm{~nm}) / \mathrm{Alq}_{3}:$ C545T (1 wt. $\%, 20 \mathrm{~nm}) / \mathrm{Alq}_{3}(45 \mathrm{~nm}) / \mathrm{LiF}(0.5 \mathrm{~nm}) / \mathrm{Al}(1 \mathrm{~nm}) / \mathrm{Ag}$ $(150 \mathrm{~nm})$ was also tested. The structure of this conventional device is slightly different from that previously reported (Ref. 7) but it will not significantly influence the device efficiency since it is a noncavity device.

The optical model used for performing the analysis adopts a full-vectorial electromagnetic approach based on the equivalence between the emission of a photon due to an electrical dipole transition and the radiation from a classical electrical dipole antenna. ${ }^{5,6,11-15}$ More details about the modeling can be found in Ref. 11 The influence of the device (microcavity) structure on the molecular transition rates (the exciton lifetimes) had been included in the electromagnetic modeling and it was found that they do not differ significantly for various microcavity structures studied in this work. The total emission intensity $I$ from the OLED as a function of the wavelength $\lambda$ and the viewing angle $\theta$ [i.e., $I(\theta, \lambda)$, with $\theta$ measured relative to the surface normal] is then obtained by averaging contributions over these distributions. Color coordinates of emission at various angles $\left[u^{\prime}(\theta)\right.$, $\left.v^{\prime}(\theta)\right]$ can then be calculated from $I(\theta, \lambda)$. To better quantify differences in colors, the $1976 u^{\prime} v^{\prime}$ uniform color space is used. $^{16}$

To better quantify influences of resonant wavelengths on emission characteristics, four parameters are defined: the outcoupling efficiency, which is the ratio of outcoupled emission to internally generated emission; the forward enhancement ratio, which is the ratio between the normal-direction luminance of a cavity device to that of an optimized conventional bottom-emitting OLED; the color shift, which is defined as [variance of $u^{\prime}(\theta)+$ variance of $\left(v^{\prime} \theta\right)$ ] and measures variation of colors over angles; and the Lambertian offset which is defined as $\Sigma \mid I(\theta) / I\left(0^{\circ}\right)$ - $\cos \theta \mid$ and measures the difference between the emission pattern of a device and the ideal Lambertian distribution [where $I(\theta)$ is spectrally integrated $I(\theta, \lambda)]$.

Along with photoluminescence (PL) of $\mathrm{Alq}_{3}$ :C545T, Figs. 2(a)-2(e) show measured (symbols) and simulated (lines) electroluminescence EL spectra with relative intensities at viewing angles of $0^{\circ}, 30^{\circ}$, and $60^{\circ}$ for devices B-F, respectively. Fairly good agreement between measured and simulated data is obtained, indicating accuracy of the simulation. In these devices, the $0^{\circ} \mathrm{EL}$ peak does shift consistently with the set $\lambda_{R}\left(0^{\circ}\right)$. For each device, the EL spectra tend to blueshift with viewing angles according to Downloaded 18 Feb 2009 to 140.112 .113 .225 . Redistribution subject
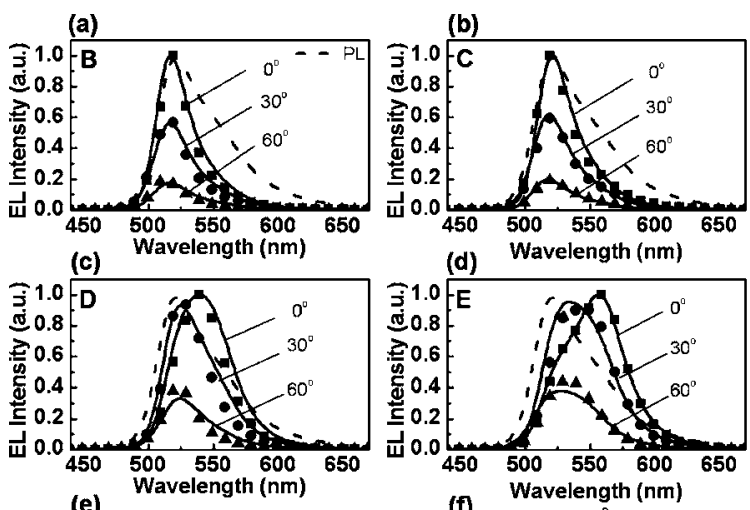

(d) Wavelength $(\mathbf{n m})$
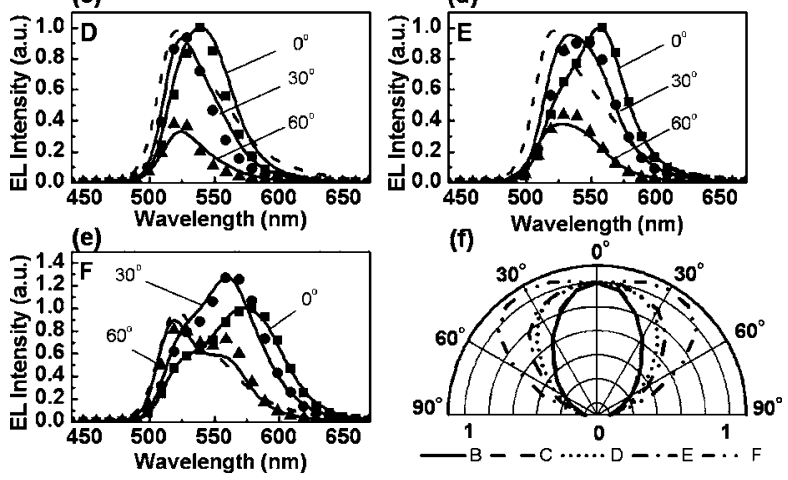

FIG. 2. Measured (symbols) and calculated (lines) EL spectra with relative intensities at viewing angles for device (a) B, (b) C, (c) D, (d) E, and (e) F. (f) Polar plots of measured EL intensities (normalized to the $0^{\circ}$ intensity) for devices B-F. In (a)-(e), PL of $\mathrm{Alq}_{3}$ : C545T is also shown for comparison.

the angular dependence of the resonant wavelength: ${ }^{17}$ $\lambda_{R}(\theta)=4 n \pi L \cos \theta /(\phi(\theta)-2 \pi m)$, where $L$ is the cavity length, $\phi(\theta)$ is total phase shift caused by reflections at two mirrors, $n$ is the refractive index of the organic material, and $m$ is the cavity mode number ( $m=0$ in the present case). The color shift with viewing angles is particularly significant for devices having $\lambda_{R}\left(0^{\circ}\right)$ set at longer wavelengths. Yet, devices having $\lambda_{R}\left(0^{\circ}\right)$ set around or smaller than $\lambda_{\mathrm{PL}}$ of C545T (e.g., B and C) exhibit hardly detectable color shift. It is mainly because the tendency of blueshift with viewing angles is largely suppressed by rapid falloff of the intrinsic emission intensity at shorter wavelengths. Figure 3(a) shows the measured color shift (symbols) as a function of $\lambda_{R}\left(0^{\circ}\right)$, which is consistent with the trend predicted by the simulation (line). For devices having $\lambda_{R}\left(0^{\circ}\right)$ set around or smaller than (a)

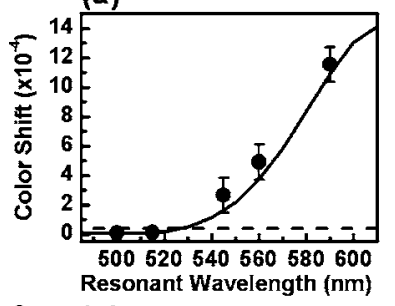

(c)

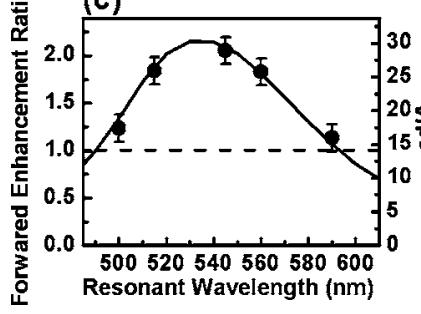

(b)

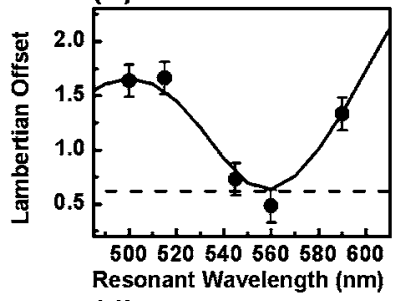

(d)

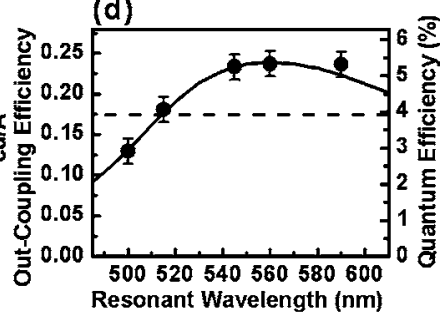

FIG. 3. Calculated values (lines) of (a) color shift, (b) Lambertian offset, (c) forward enhancement ratio, and (d) outcoupling efficiency as a function of the normal-direction resonant wavelength. The calculated results are compared with experimentally obtained values (symbols) of color shift, Lambertian offset, cd/A efficiency, and external quantum efficiency in (a)-(d), respectively. The horizontal dashed lines in (a)-(d) represent experimental values of the conventional bottom-emitting device A.

o AIP license or copyright; see http://apl.aip.org/apl/copyright.jsp 


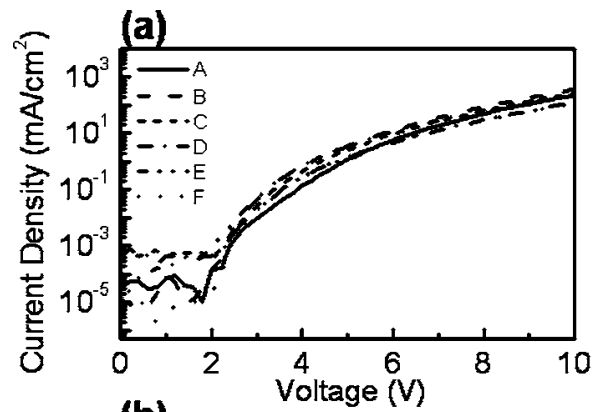

(b)

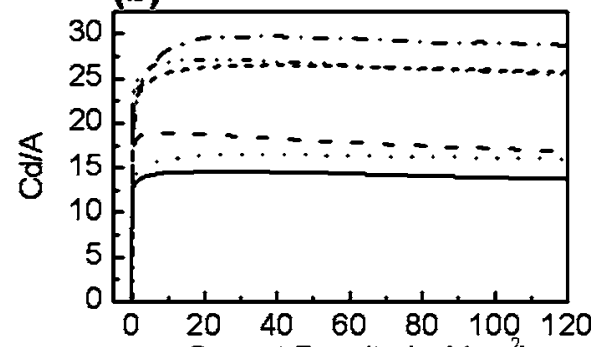

(c)

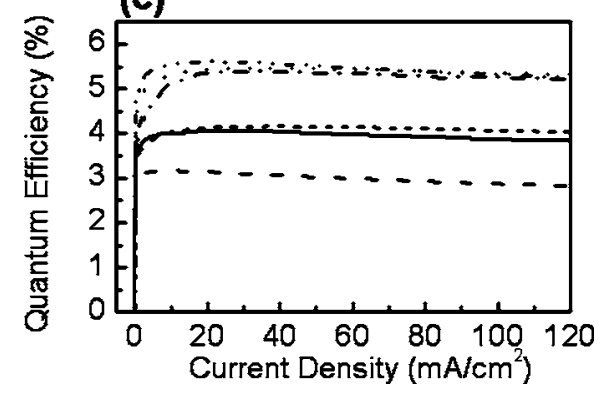

FIG. 4. (a) $I-V$ characteristics, (b) external EL quantum efficiencies, and (c) $\mathrm{cd} / \mathrm{A}$ efficiencies of devices A-F.

$\lambda_{\mathrm{PL}}$, the values of color shift are comparable to that of the conventional device A $\left(\sim 4 \times 10^{-5}\right.$, the horizontal dashed line).

Figure 2(f) compares angular distributions of the measured EL intensity (spectrally integrated and normalized to $0^{\circ}$ intensity) for devices B-F,. Devices having $\lambda_{R}\left(0^{\circ}\right)$ around or smaller than $\lambda_{\mathrm{PL}}$ (e.g., $\mathrm{B}$ and $\mathrm{C}$,) show strongly directed emission toward the surface normal. Devices having $\lambda_{R}\left(0^{\circ}\right)$ 20-40 nm longer than $\lambda_{\mathrm{PL}}$ (e.g., D and E) show emission patterns close to the Lambertian distribution. For the device having $\lambda_{R}\left(0^{\circ}\right)$ substantially longer than $\lambda_{\mathrm{PL}}$ (e.g., F), the emission pattern shows the highest intensity at an off-axis angle. Since $\lambda_{R}(\theta)$ in general blueshifts with viewing angles, the emission pattern is controlled not only by the microcavity modification but also by the spectral shape of the intrinsic PL. As such, the directionality of microcavity emission is enhanced when setting $\lambda_{R}\left(0^{\circ}\right)$ at the short-wavelength falling edge of the PL spectrum; setting $\lambda_{R}\left(0^{\circ}\right)$ at the longwavelength falling edge makes the strongest emission occur at some off-axis angle; setting $\lambda_{R}\left(0^{\circ}\right)$ somewhere in between may give nearly Lambertian distribution. Figure 3(b) shows the measured Lambertian offset (symbols) as a function of $\lambda_{R}\left(0^{\circ}\right)$, which is consistent with the trend predicted by the simulation (line). For devices having $\lambda_{R}\left(0^{\circ}\right)$ set $20-40 \mathrm{~nm}$ longer than $\lambda_{\mathrm{PL}}$ (e.g., $\mathrm{D}$ and $\mathrm{E}$ ), the Lambertian offset achieves a lowest value similar to that of the conventional device A (0.6, the horizontal dashed line).

Figure 4(a) shows the current-voltage (I-V) characteristics of devices A-F. Electrical properties of these devices do Downloaded 18 Feb 2009 to 140.112.113.225. Redistribution subject to AIP license or copyright; see http://apl.aip.org/apl/copyright.jsp not much differ ensuring that experimentally observed large variations in emission characteristics can be attributed mainly to optical microcavity effects rather than other electrical effects (e.g., moving of the carrier recombination zone due to altered carrier injection, degraded balance in carrier injection, etc.). As shown in Fig 4(b) and Fig. 4(c), both measured cd/A efficiency (at the normal direction) and quantum efficiency vary substantially among devices. As shown in Figs. 3(c) and 3(d), the measured cd/A efficiencies and quantum efficiencies (symbols) as a function of $\lambda_{R}\left(0^{\circ}\right)$ follow closely the calculated forward enhancement ratio [Fig. 3(c)] and the calculated outcoupling efficiency [Fig. 3(d)], respectively. As expected, setting $\lambda_{R}\left(0^{\circ}\right)$ near $\lambda_{\mathrm{PL}}$ (e.g., $515 \mathrm{~nm}$ for $\mathrm{C}, 545 \mathrm{~nm}$ for $\mathrm{D}$ ) gives the highest forward enhancement ratio of $\sim 2(26.3 \mathrm{~cd} / \mathrm{A}$ of $\mathrm{C}$ and $29.6 \mathrm{~cd} / \mathrm{A}$ of D versus $14.6 \mathrm{~cd} / \mathrm{A}$ of the conventional device A). On the other hand, by setting $\lambda_{R}\left(0^{\circ}\right) 20-40 \mathrm{~nm}$ longer than $\lambda_{\mathrm{PL}}$ (e.g., $545 \mathrm{~nm}$ for $\mathrm{D}, 560 \mathrm{~nm}$ for E), the highest outcoupling and quantum efficiency are achieved $(\sim 1.4$ times higher than the conventional bottom-emitting device, e.g., $5.4 \%$ of $\mathrm{D}$ and $5.6 \%$ of $\mathrm{E}$ versus $4 \%$ of $\mathrm{A}$ ).

In conclusion, the emission characteristics of microcavity OLEDs show strong dependence on the resonant wavelength. In addition, there are trade-offs between different characteristics in choosing the resonant wavelength, as clearly manifested in Fig. 3. Thus the exact design of the microcavity device would depend on actual applications. For display applications that desire small color shifts over viewing angles and highest brightness along the normal viewing direction, $\lambda_{R}\left(0^{\circ}\right)$ can be set near $\lambda_{\mathrm{PL}}$. For applications (such as lighting) in which highest possible external quantum efficiencies and more uniform intensity distributions are highly desired while the color shift may be more tolerable, $\lambda_{R}\left(0^{\circ}\right)$ may be moved 20-40 nm away from $\lambda_{\mathrm{PL}}$.

The authors would like to acknowledge financial support from National Science Council of Republic of China. One of the authors (C.-L.L.) is also grateful for financial support from MediaTek Fellowship.

${ }^{1}$ N. Takada, T. Tsutsui, and S. Saito, Appl. Phys. Lett. 63, 2032 (1993).

${ }^{2}$ A. Dodabalapur, L. J. Rothberg, R. H. Jordan, T. M. Miller, R. E. Slusher, and J. M. Phillips, J. Appl. Phys. 80, 6954 (1996).

${ }^{3}$ M.-H. Lu, M. S. Weaver, T. X. Zhou, M. Rothman, R. C. Kwong, M. Hack, and J. J. Brown, Appl. Phys. Lett. 81, 3921 (2002).

${ }^{4}$ H. Riel, S. Karg, T. Beierlein, W. Rieß, and K. Neyts, J. Appl. Phys. 94, 5290 (2003).

${ }^{5}$ C.-W. Chen, P.-Y. Hsieh, H.-H. Chiang, C.-L. Lin, H.-M. Wu, and C.-C. Wu, Appl. Phys. Lett. 83, 5127 (2003).

${ }^{6}$ C.-W. Chen, C.-L. Lin, and C.-C. Wu, Appl. Phys. Lett. 85, 2469 (2004).

${ }^{7}$ C.-L. Lin, H.-W. Lin, and C.-C. Wu, Appl. Phys. Lett. 87, 021101 (2005).

${ }^{8}$ Q. Huang, K. Walzer, M. Pfeiffer, and K. Leo, J. Appl. Phys. 100, 064507 (2006).

${ }^{9}$ L. S. Hung, C. W. Tang, M. G. Mason, P. Raychaudhuri, and J. Madathil, Appl. Phys. Lett. 78, 544 (2001).

${ }^{10}$ X. Zhou, M. Pfeiffer, J. Blochwitz, A. Werner, A. Nollau, T. Fritz, and K. Leo, Appl. Phys. Lett. 78, 410 (2001).

${ }^{11}$ C.-L. Lin, T.-Y. Cho, C.-H. Chang, and C.-C. Wu, Appl. Phys. Lett. 88, 081114 (2006).

${ }^{12}$ W. Lukosz, J. Opt. Soc. Am. 67, 1607 (1977).

${ }^{13}$ J. E. Sipe, Surf. Sci. 105, 489 (1981).

${ }^{14}$ K. Neyts, J. Opt. Soc. Am. A 15, 962 (1998).

${ }^{15}$ W. L. Barnes, J. Mod. Opt. 47, 725 (2000).

${ }^{16}$ Color in Electronic Displays, edited by H. Widdel and D. L. Post (Plenum, New York, 1992), 3, p. 39.

${ }^{17}$ M. Born and E. Wolf, Principles of Optics, 7 th ed. (Cambridge University Press, Cambridge, 1999), 387. 